\title{
Botulinum toxin treatment for lower limb extensor spasticity in chronic hemiparetic patients
}

\author{
S Hesse, D Lücke, M Malezic, C Bertelt, H Friedrich, M Gregoric, K H Mauritz
}

Klinik Berlin,

Department of

Neurological

Rehabilitation, Free

University of Berlin,

Germany

S Hesse

D Lücke

M Malezic

C Bertelt

H Friedrich

K H Mauritz

Rehabilitation

Institute, Ljubljana,

Slovenia

M Gregoric

Correspondence to:

Dr $S$ Hesse, Klinik Berlin

Department of Neurologica

Rehabilitation, Fre

University Berlin, Kladower

Damm 223, 14089 Berlin, Germany.

Received 4 February 1994

and in revised form

12 April 1994.

Accepted 16 May 1994

\begin{abstract}
Twelve chronic hemiparetic outpatients with pronounced lower limb extensor spasticity were injected with 400 units of botulinum toxin A, EMG guided into the soleus, tibialis posterior, and both heads of the gastrocnemius muscles. Botulinum toxin $A$ caused a definite reduction of plantar flexor spasticity, in 10 patients two weeks after the injection, as assessed by the Ashworth scale. Four of the patients were able to achieve active dorsiflexion of their affected ankle. Gait analysis including the measurement of vertical ground reaction forces showed a statistically significant $(p<0.01)$ improvement in velocity, stride length, stance symmetry, and the length of the force point of action under the affected foot. Qualitative improvements on the force diagrams indicated a better loading, advancement of the body, and push off of the affected limb in seven patients. Eight weeks after the injection the effects waned.
\end{abstract}

(F Neurol Neurosurg Psychiatry 1994;57:1321-1324)

Botulinum toxin A injection has been successfully used in the treatment of focal dystonias. ${ }^{1}$ Early studies on the treatment of spasticity reported a significant reduction in tone of the leg adductors, upper limb flexor spasticity, and spastic foot drop. ${ }^{2-5}$ One report included an assessment of the ankle range of motion and clinical observation of gait four weeks after the injection of the British product. ${ }^{4}$ Problems associated with plantar flexor spasticity during gait are a forefoot contact, a reduced loading and stance duration on the affected limb, a stance equinus, a poor

Table 1 Patient data and Ashworth grades

\begin{tabular}{|c|c|c|c|c|c|}
\hline \multirow[b]{2}{*}{ Subject } & \multirow[b]{2}{*}{ Age/sex } & \multirow{2}{*}{$\begin{array}{l}\text { Stroke } \\
\text { (months) }\end{array}$} & \multicolumn{3}{|c|}{ Ashworth scale } \\
\hline & & & Before & 2 weeks & 8 weeks \\
\hline $\begin{array}{l}1 \\
2 \\
3 \\
4 \\
5 \\
6 \\
7 \\
8 \\
9 \\
10 \\
11 \\
12\end{array}$ & $\begin{array}{l}57 / M \\
59 / M \\
52 / M \\
43 / M \\
57 / M \\
34 / F \\
30 / M \\
53 / M \\
51 / M \\
48 / F \\
49 / M \\
46 / \mathrm{F}\end{array}$ & $\begin{array}{l}6 \cdot 7 \\
11 \\
19 \\
13 \cdot 3 \\
57 \\
12 \\
84 \\
19 \\
36 \\
18 \\
7 \cdot 2 \\
11\end{array}$ & $\begin{array}{l}4 \\
4 \\
4 \\
5 \\
4 \\
3 \\
5 \\
5 \\
5 \\
5 \\
4 \\
5 \\
4\end{array}$ & $\begin{array}{l}3 \\
3 \\
3 \\
4 \\
4 \\
3 \\
4 \\
3 \\
4 \\
3 \\
3 \\
3\end{array}$ & $\begin{array}{l}3 \\
4 \\
2 \\
5 \\
4 \\
3 \\
5 \\
4 \\
5 \\
3 \\
4 \\
3\end{array}$ \\
\hline
\end{tabular}

Ashworth grades (0-5) tested for ankle dorsiflexion of the affected lower limb.

progression of the body with reduced stride length, a lack of push off, and a dragging of the toes. ${ }^{6}$

To elucidate further the effects of botulinum treatment on lower limb extensor spasticity, we conducted a study in chronic hemiparetic patients, injecting a larger dose of the United States product than reported previously.

\section{Methods}

SUBJECTS (TABLE 1)

Twelve chronic hemiparetic outpatients (three women, nine men), who gave written informed consent, participated in the study. Their mean age was $48 \cdot 2$ (range 30-59) years. Eight had a right and four a left hemiparesis with a mean stroke interval of 24.5 (range 6.7-84) months. Aetiology was in all cases a supratentorial lesion with ischaemia in eight and a vascular haemorrhage in four patients.

All had a pronounced increase in muscle tone of the lower limb. One patient scored grade 3 (large increase in muscle tone), six scored grade 4 (passive movements difficult), and five scored grade 5 (rigid) on the modified Ashworth scale, tested during passive ankle dorsiflexion in a supine position. Walking barefoot, initial contact was made with the forefoot and gait was impaired by stance equinus in all cases. They could walk independently at least 10 metres with the help of devices: all except one walked with a cane and eight of them wore a semirigid ankle and foot orthosis.

Before their admission into this study, all participants had been trained within intensive stroke rehabilitation inpatient programmes including physiotherapy, oral antispastic medication, icing, and application of serial castings in three of them.

\section{INJECTION TECHNIQUE}

Botulinum toxin A (Botox, Allergan) was injected through 21 gauge needles that were Teflon coated except for the tip; these were also used as EMG electrodes. Botulinum toxin $\mathrm{A}$, diluted with saline to a concentration of 10 units $/ 0 \cdot 1 \mathrm{ml}$, was injected at two sites close to the motor point. The point was identified by standard neurophysiological techniques. The toxin was injected only when either a continuous or stretch induced EMG activity was recorded, otherwise another injection site in the vicinity was checked. In all patients the soleus, tibialis posterior, and medial and lateral head of the gastrocnemius 
Table 2 Gait variables

\begin{tabular}{lccc}
\hline & Before & 2 weeks & 8 weeks \\
\hline Velocity (m/s) & $0 \cdot 30(0 \cdot 15)$ & $0 \cdot 38(0 \cdot 17)^{\star}$ & $0 \cdot 35(0 \cdot 18)$ \\
Cadence (steps/min) & $61 \cdot 9(19 \cdot 2)$ & $67 \cdot 8(18 \cdot 5)$ & $68 \cdot 3(20 \cdot 0)$ \\
Stride (m) & $0 \cdot 59(0 \cdot 21)$ & $0 \cdot 70(0 \cdot 22)^{\star}$ & $0 \cdot 60(0 \cdot 22)$ \\
Stance symmetry (\%) & $68 \cdot 7(21 \cdot 8)$ & $77 \cdot 1(17 \cdot 3)^{\star}$ & $73 \cdot 8(13 \cdot 5)$ \\
Swing symmetry (\%) & $68 \cdot 1(17 \cdot 0)$ & $69 \cdot 6(19 \cdot 7)$ & $64 \cdot 7(14 \cdot 3)$ \\
DS init (\%) & $31 \cdot 5(16 \cdot 9)$ & $24 \cdot 7(10 \cdot 7)$ & $22 \cdot 0(7 \cdot 1)$ \\
DS term (\%) & $23 \cdot 2(8 \cdot 9)$ & $21 \cdot 5(7 \cdot 1)$ & $20 \cdot 1(5 \cdot 7)$ \\
Traj-aff (\%) & $40 \cdot 7(9 \cdot 7)$ & $48 \cdot 4(10 \cdot 8)^{\star}$ & $49 \cdot 9(9 \cdot 1)^{\star}$ \\
Traj-non-aff (\%) & $61 \cdot 6(11 \cdot 3)$ & $66 \cdot 6(10 \cdot 3)$ & $65 \cdot 4(11 \cdot 3)$ \\
\hline
\end{tabular}

Results are means (SD); stance symmetry, swing symmetry = ratio of the phase; DS init, DS term $=$ double stance durations, initial with the affected leg in front and terminal with the affected leg behind, normalised by stride duration; Traj-aff, Traj-non-aff = length of the trajectories of the force point of action under the affected and non-affected foot normalised by foot length.

$\star$ Significant improvement at $\mathrm{p}<0.01$.

muscles of the affected side were treated with a total dose of 400 units of Botox (100 units ( $=$ $1 \mathrm{ml}$ of fluid) each). The rather high amount of toxin was derived from our experience with the treatment of severe upper limb flexor spasticity, ${ }^{5}$ where a dosage of at least $40 \mathrm{ng}$ of the British product (equivalent to about 320 mouse units of the United States product, personal communication, Allergan) proved to be effective.

\section{MEASUREMENTS}

The patients were examined by two independent persons before the treatment, and two and eight weeks after it. Muscle tone was rated by the modified Ashworth scale. ${ }^{7}$ The ankle range of motion was tested in a supine position for dorsiflexion and eversion. It was graded from 0 for no increase in muscle tone to 5 for a rigid plantar flexion and inversion.

The Rivermead score for the leg and trunk was used to assess motor function after a stroke. ${ }^{8}$

An Infotronic force shoe system was used for the kinesiological measurements during gait with preferred shoes and walking canes but without ankle and foot orthosis. ${ }^{9}$ Velocity, cadence, stride length, stance, and swing time symmetry, double stance durations (initial with the affected leg in front and terminal with the affected leg behind), were used for statistical analysis. Trajectories of the force point of action under both feet were also assessed in \% of the foot length. The nonparametric Wilcoxon test was used for the statistical analysis (corrected $a=0.01$ ).

\section{Results}

Two weeks after the injection 11 of the 12 patients reported an improvement: eight could walk better, achilles tendon clonus was diminished in six, and the toe clawing within the extensor synergy was reduced in five patients. An ankle and foot orthosis was no longer used by one patient and was used to a lesser extent in four. Eight weeks after the injection six of the 11 patients with subjective improvement after two weeks reported a diminished effect of the treatment. All but one patient then used an ankle and foot orthosis as before the injection.

ASHWORTH SCALE (TABLE 1)

Two weeks after the injection the Ashworth spasticity scale showed a reduction of 1 point in eight and a reduction of 2 points in two patients when tested for ankle dorsiflexion. Ankle eversion was reduced by 1 point in eight patients at the same time.

Eight weeks after the injection, the Ashworth scale showed a fading of the effects in six patients by 1 point in ankle dorsiflexion, to the previous level in four, and a partial fading in two. They had initial grades of 5 (five patients) and 4 (one patient). The dorsiflexion remained unchanged in three patients and further improved in one. These patients had an initial Ashworth grade 4. At the same time the improvement of eversion remained stable in five patients.

\section{RIVERMEAD SCORE}

Two weeks after the injection the Rivermead score improved by 1 point in three patients and by 2 points in one patient. Two of these four patients could then achieve dorsiflexion of the ankle with the knee partly flexed and another two could achieve this with an extended knee. One patient could additionally tap with the non-affected foot five times while standing on the affected foot. Eight weeks later these effects could still be seen in two patients.

\section{GAIT ANALYSIS (TABLE 2)}

Two weeks after the injection, velocity, stride length, stance symmetry, and length of the trajectory of the force point of action under the affected foot significantly improved (fig 1). Cadence, double support times, and the trajectory under the non-affected foot also improved but did not reach the chosen significance level of $p=0 \cdot 01$. The beginning of the force trajectories under the affected foot showed initial contact with the flat foot instead of the forefoot in nine patients. Vertical mean force diagrams, averaged over the four consecutive trials, showed better loading and push off of the affected limb in seven patients two weeks after the injection, indicated by a steeper ascent and descent of the force curves (fig 2). The variability of the forces was also reduced.

Eight weeks after the injection, only the trajectory of the force point of action remained significantly longer than before injection. In six patients the initial contact was still made with the flat foot. Velocity, stride length, and stance symmetry did not differ significantly. The qualitative changes in force diagrams (better loading, push off, and less variability of the curves) remained stable in two out of seven patients.

Figure 1 gives the trajectories of a right hemiparetic patient before and two weeks after injection. Before treatment there was an initial contact with the forefoot of the affected leg, then the point of action moved towards the midfoot and again forward during the terminal stance phase. The trajectory remained under the forefoot throughout the stance phase and did not exceed $40 \%$ of the foot length. Due to the ankle plantar flexion of the affected leg the patient had to lean forward 
Figure 1 Trajectories of the force point of action under the non-affected and affected foot in a right hemiparetic patient before and two weeks after the injection of botulinum toxin $A$.

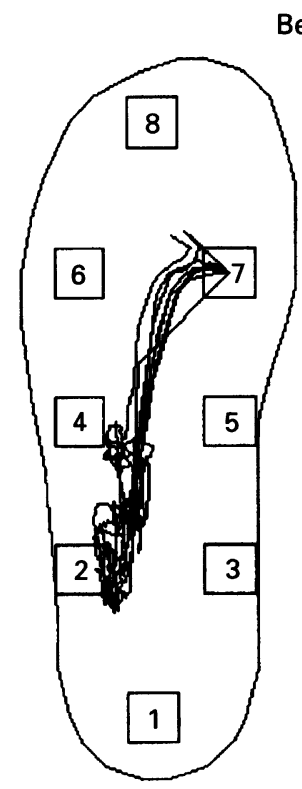

Non-affected
Before

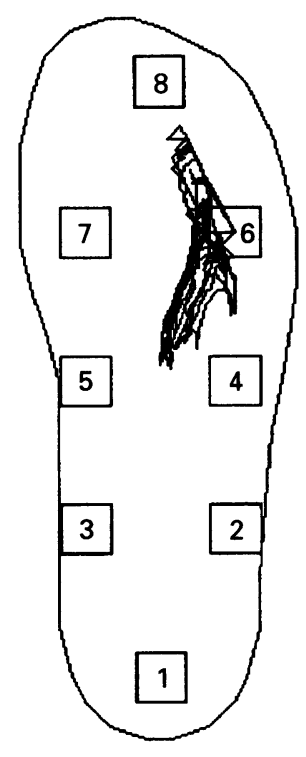

Affected

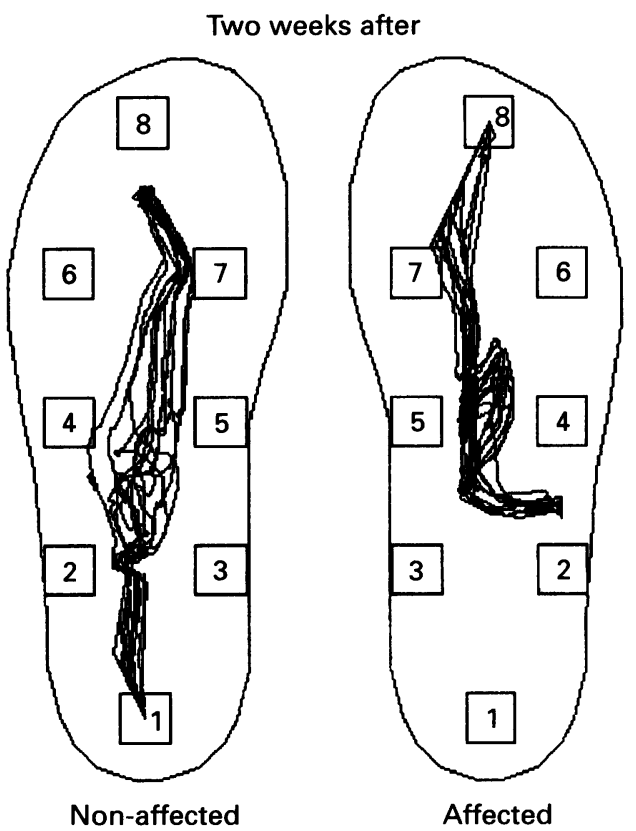

while the hip joints remained flexed. Therefore he loaded the non-affected leg at the midfoot. After the injection there was an initial contact with the lateral border of the foot immediately followed by the flat foot. The force point of action moved forward from the rear part to about $60 \%$ of the foot length. The force trajectory also improved on the non-affected side.

Figure 2 gives mean (SD) forces for the patient for whom data are presented in fig 1 . Two weeks after the injection better loading and push off were displayed together with considerable reduction of variability. Similar but less expressed improvement also occurred on the non-affected side. The effects shown in figs 1 and 2 were diminished after eight weeks.

\section{SIDE EFFECTS}

There were no systemic side effects. Locally, botulinum toxin A caused slight weakness of the plantar flexion and knee extension in two patients two weeks after the injection. Eight weeks after the treatment the paresis was no longer seen in these two patients.

\section{Discussion}

Muscle tone, gait ability, and other motor functions improved to some extent in all patients after the injection of botulinum toxin A. Clinically, achilles tendon clonus and painful toe clawing were reduced and the patients reported better gait. These improvements could be ascribed to the treatment with botulinum toxin $\mathrm{A}$ as the muscle tone had not changed appreciably despite the fact that all patients had received an intensive stroke rehabilitation inpatient programme before the injection. A mean stroke interval of 24.5 months excluded confounding effects of spontaneous recovery. ${ }^{10}$ Also, the patients did not receive any intensive physiotherapy during the study; the treatment did not exceed three periods of 30 minutes per week. reduce muscle tone as indicated by the
Botulinum toxin $\mathrm{A}$ has been shown to
Figure 2 Vertical force diagrams of the nonaffected and affected lower limb in a right hemiparetic patient before and two weeks after the injection of botulinum toxin $A$.
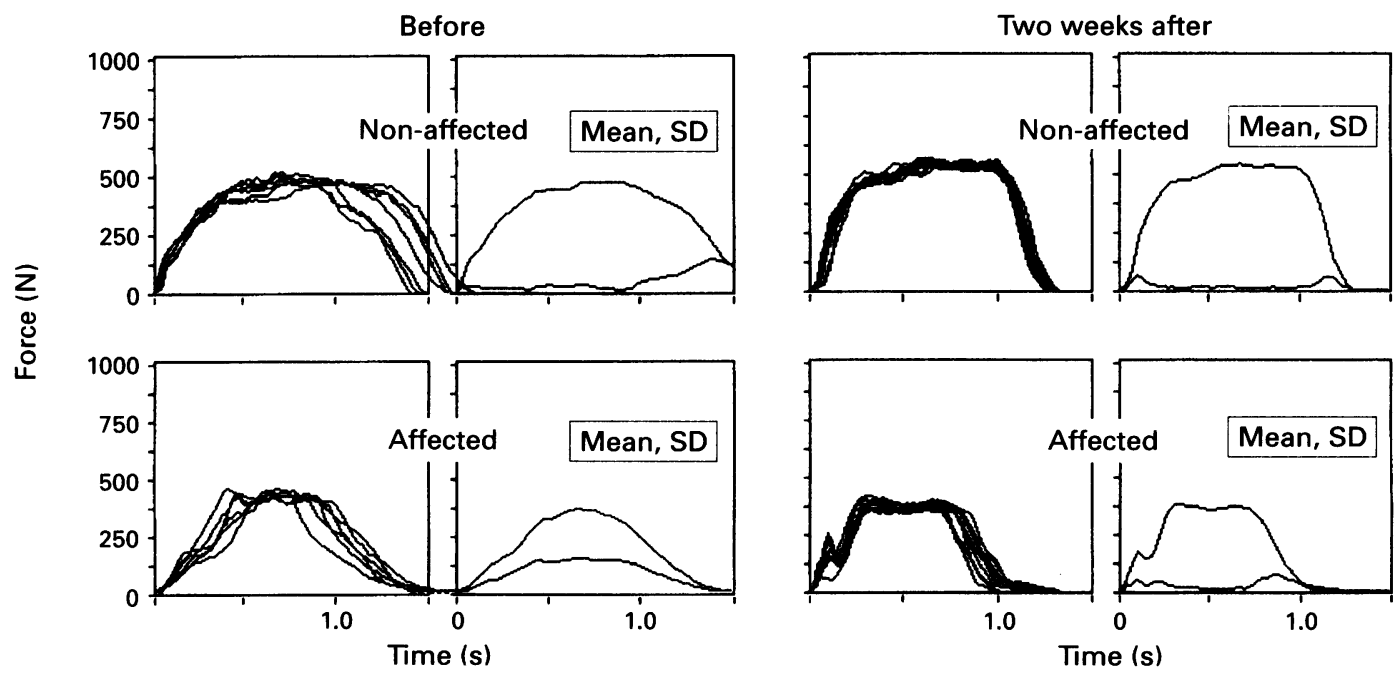
Ashworth grades of ankle dorsiflexion and eversion. ${ }^{2-5}$ With the reduction in our patients, gait velocity notably improved with a mean of $27 \%$ for the whole group of patients, exceeding a reported $25 \%$ threshold of clinical significance. ${ }^{11}$ The increase in gait velocity resulted from a longer stride length, whereas cadence remained stable. The improvements were noted in the patients with reduced ankle muscle tone.

Plantar-flexor spasticity mainly reduces stride length by an impaired body advancement during the stance phase. ${ }^{6}$ Correspondingly, the length of the force trajectories under both feet $(40.7 \%$ for the affected and $61.3 \%$ for the non-affected leg) was reduced. Normal values range from $85 \%$ to $90 \%$ of the foot length. ${ }^{12}$

The impaired forward movement of the lower leg is partly explained by a premature calf muscle activation already in the terminal swing phase. ${ }^{1314}$ It leads to a tension increase sufficiently large to shorten the muscles before the body has passed ahead of the foot. An initial forefoot contact in combination with a lowered stretch threshold of the spastic plantar flexor muscles promotes this concentric muscle activation. ${ }^{13}$ By lowering muscle tone, botulinum toxin might have increased the stretch threshold and thus improved the progression of body and stride length. This assumption is supported by the six patients who reported that achilles tendon cloni were lessened. The change from initial forefoot contact to foot flat with the affected leg in nine patients further supported body progression.

Premature and persistent soleus activity can also cause stance equinus, which in turn leads to poor weight acceptance, asymmetry of the stance, and double support phases. ${ }^{15}$ The vertical force diagrams and the stance symmetry ratios displayed an improvement of the stance equinus and weight acceptance two weeks after the injection, which lowered the risk of spraining an ankle.

Besides gait, other motor functions also improved as confirmed by the Rivermead scores. Four patients could achieve dorsiflexion of their affected ankle while lying down. Botulinum toxin A injected into the plantar flexors may have reduced reciprocal inhibition caused by the Ia extensor afferents, which inhibit flexor motor neurons in spastic lower limbs. ${ }^{16} 17$

Eight weeks after the injection the muscle tone increased again, partly in two patients and completely in four. In the four patients with complete increase of muscle tone after eight weeks, three initially had a rigid ankle in plantar flexion (Ashworth grade 5), and one had a considerably increased muscle tone with difficult passive movement (Ashworth grade
4). The two patients with a partial increase had an initial grade of 5 . It seemed that the effects lasted for the shortest duration in the most spastic patients. The alteration of mechanical properties of the muscle, influenced by the poststroke period, might also shorten the effects. ${ }^{18}$

Despite the increase of muscle tone, the force trajectory under the affected foot remained significantly longer during gait $(\mathrm{p}<$ 0.01 ), even in four patients with increased spasticity. The gait cadence also remained high, implying the value of better and more frequent gait practice during the study. As the patients were not extensively treated during the eight week period, a comprehensive rehabilitation programme might preserve the effects of injection.

So far, the high costs of the botulinum toxin A injection and possible short term effect raises problems for its application. A double blind placebo control study should quantify the effects and validate the cost effectiveness of the botulinum toxin treatment.

The study was supported by a grant of the Maria-Sonnenfeld Gedächtnisstiftung. Botulinum toxin A was supplied by Allergan Company.

1 American Academy of Neurology. Assessment: the clinical usefulness of botulinum toxin-A in treating neurological disorders. Neurology 1990;40:1332-6.

Das TK, Park DM. Botulinum toxin in treating spasticity. Br f Clin Prod 1989;43:401-2.

3 Snow BJ, Tsui JL, Bhatt MH, Varelas M, Hashimoto SA, Clane DB. Treatment of spasticity with botulinum toxin: a double blind study. Ann Neurol 1990;28:512-5.

4 Dengler $R$, Neyer U, Wolfarth $K$, Bettig U, Janzik $H H$ Local botulinum toxin in the treatment of spastic drop foot. $\mathcal{F}$ Neurol 1992;239:375-8.

5 Hesse S, Friedrich H, Domasch C, Mauritz KH. Botulinum toxin therapy for upper limb flexor spasticity: Botulinum toxin therapy for upper limb flexor spasticity: preliminary result

6 Perry J. Gait analysis. Thorofare: Slack Inc, 1992:312-27. Bohannon RW, Smith MB. Interrater reliability of a modified Ashworth spastic scale of muscle spasticity. Physical Therapy 1987;67:206-7.

8 Lincoln N, Leadbitter D. Assessment of motor function in stroke patients. Physiotherapy 1979;65:48-51.

9 Hermens HJ, deWaal CA, Buurke J, Zilvold G. A new gait analysis system for clinical use in a rehabilitation center. Orthopedics 1986;9:1669-75.

10 Skilbeck CE, Wade DT, Hewer RL, Wood VA. Recovery fter stroke. F Neurol Neurosurg Psychiatry 1983;46:5-8.

11 Collen FM, Wade DT, Bradshaw CM. Mobility after stroke: reliability of measures of impairment and disability. International Disabilities Studies 1990;12:6-9.

12 Seichert N, Senn E. Clinic gait analysis with force plates including double-stride-parameters. In: Brandt $\mathrm{T}$, including double-stride-parameters. In: Brandt T, Paulus W, Bles W, Dietrich M, Krafczyk S, Straube A,

13 Knuttson E, Richards C. Different types of disturbed motor control in gait of hemiparetic patients. Brain 1979;102:405-30.

14 Shiavi R, Bugle HJ, Limbrid T. Electromyographic gait assessment, part 2: preliminary assessment of hemiparetic synergy patterns. $\mathcal{f}$ Rehabil Res Dev 1987;24: 24-30.

15 Perry J, Waters R, Perrin T. Electromyographic analysis of equinovarus following stroke. Clin Orthop 1978;131: $47-53$.

16 Yanagisawa N, Tanaka R, Ito Z. Reciprocal Ia inhibition in spastic hemiplegia of man. Brain 1976;99:555-74.

17 Artieda J, Quesada P, Obeso JA. Reciprocal inhibition between forearm muscles in spastic hemiplegia. Neurology 1991;41:286-9.

18 Thilmann AF, Fellows SJ, Garms E. The mechanism of spastic muscle hypertonus. Brain 1991;114:233-44. 